\title{
CONVERSION OF FIELD IONIZATION KINETICS DATA TO UNIMOLECULAR RATE CONSTANT FUNCTIONS. A SIMPLE NUMERICAL APPROACH
}

\author{
WILLI A. BRAND *, JOCHEN STOCKLÖV and HANS JÖRG WALTHER ** \\ Institut für Physikalische Chemie, Universität Bonn, 5300 Bonn I (F.R.G.)
}

(First received 24 August 1983; in final form 30 January 1984)

\section{ABSTRACT}

A method has been developed to convert experimental $k(t)$ vs. $t$ functions, measured in a field ionization kinetics (FIK) experiment into a table of unimolecular rate constants, $k(E)$, active in the dissociation and their relative contributions. By assuming a certain form of the internal energy distribution function, $P(E)$, of the precursor ion, the $k(E)$ values are related to the excess energy scale through knowledge of the lowest decomposition energy threshold and the measured total amount of fragmentation.

The method is applied to the halogen loss from three halobenzene molecular ions. Satisfactory agreement with literature data is found when a smooth function with energy is used for the internal energy distribution.

The problems involved in this kind of analysis are discussed for the methyl loss from tert-butylbenzene precursor ion. A change in slope in the $k(t)$ function is observed experimentally. Possible explanations for such an observation are discussed. In the present case, it is probably due to a structured internal energy distribution.

\section{INTRODUCTION}

It is commonly accepted that the theory of mass spectra (RRKM [1,2], QET $[3,4]$ ) governs the fragmentation of ions irrespective of ion preparation. The decay of parent ions is described through individual rate constants, $k(E)$, characteristic for one reaction channel. In its simplest form, $k(E)$ is given by

$k(E)=\frac{\sigma}{h} \frac{W^{+}\left(E-E_{0}\right)}{\rho(E)}$

The important variable is the internal energy, $E$, sometimes referred to as the

* To whom correspondence should be addressed. Present address: VG Instruments, Stresemannring 12-16, 6200 Wiesbaden, F.R.G.

** Present address: Zentrale Funktion Forschung, Ciba Geigy AG, CH-4002 Basel, Switzerland. 
excess energy beyond the ionization threshold. $\sigma$ is the reaction degeneracy factor, $h$ is Planck's constant, $\rho(E)$ the density of states of the precursor ion at energy $E$ and $W^{+}\left(E-E_{0}\right)$ is the sum of states of the transition state beyond the decomposition threshold $E_{0} . W^{+}\left(E-E_{0}\right)$ and $\rho(E)$ may be calculated using the normal frequencies of the species as a measure of structure and counting the states. Alternatively, sufficiently accurate approximations are available. The theory provides rate constants between a minimum derived from Eq. (1)

$k\left(E_{0}\right)=\frac{\sigma}{h \rho\left(E_{0}\right)}$

for $W^{+}=1$ and a maximum rate, reasonably up to $10^{14} \mathrm{~s}^{-1}$. The latter is given by the time of one vibrational period of the bond to be broken.

In contrast to the large dynamic range of rate constants provided by the theory, there is only a small range of $k(E)$ values accessible through experiments. In ionic dissociations, internal energy selected species are monitored in two experiments, photoelectron photoion coincidence spectroscopy (PEPICO [5]) and charge exchange (CE [6]). Both experiments cover a range of $k(E)$ between $10^{4}$ and $5 \times 10^{6} \mathrm{~s}^{-1}$. Below $10^{4} \mathrm{~s}^{-1}$, the signal intensity is too low and beyond $5 \times 10^{6} \mathrm{~s}^{-1}$, the time resolution is exceeded. The rate constants of interest in a mass spectrometer, however, extend to much higher values. They depend on the energy available for dissociation. In electron impact (EI), this energy is of ten large enough to cause dissociation within one vibrational period. From this fact arises the necessity to develop experimental methods capable of determining rate constants larger than $5 \times 10^{6} \mathrm{~s}^{-1}$.

Recently, it has been shown that field ionization kinetics (FIK) is capable of extracting approximate $k(E)$ functions from experimental data [7]. This was the first step towards an experimental determination of large rate constants. The method suffers from a number of drawbacks. Among these are that FIK is not an experiment employing internal energy selection and that the analysis is restricted to parent ion decays with one decomposition channel only. In this paper, we present a simple numerical approach to the determination of $k(E)$ from FIK data. It does not suffer some of the restrictions necessary in the more exact method of our previous work. In addition, we want to comment on the general applicability of the FIK method and its usefulness in rate constant determination.

\section{PRINCIPLES OF THE METHOD}

Figure 1 shows schematically the typical peak shapes observed in a field ionization experiment when using a magnetic sector single focusing mass 


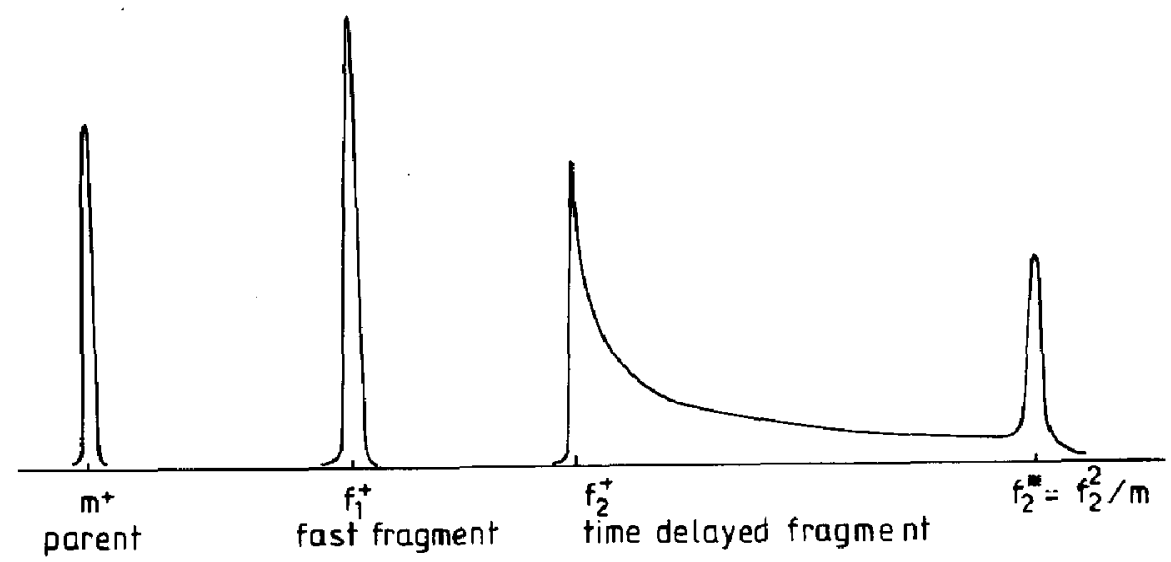

Fig. 1. Typical peak shapes observed in field ionization in a single focusing magnetic sector instrument. The asymmetric peak tailing of $f_{2}^{+}$is converted to a time scale.

spectrometer. Because of the precise definition of the ionization zone in field ionization, the kinetic energy spread of the parent ion is a couple of volts only at the high field strengths used. Fragment ions due to field dissociation [8] and such with decay rate constants larger $10^{12} \mathrm{~s}^{-1}$ are also recorded as sharp, symmetric signals. The region of interest in the spectrum for FIK is the asymmetric tailing of the fragment ions towards the metastable peak position at $\mathrm{f}_{2}^{2} / \mathrm{m}$ (for the counterelectrode held on ground potential) and the metastable itself. The latter may be split through introducing cylinders behind the counterelectrode which are kept at a potential different from ground level. The tailing is due to dissociations taking place between the field anode and the counterelectrode, representing the first ten nanoseconds following ionization. The time resolution is as short as $10^{-11} \mathrm{~s}$, depending on the mass resolution in a single focusing mass spectrometer. Provided the potential distribution between emitter and counterelectrode is known with sufficient accuracy, the mass scale can be converted to a time scale by solving the equation of motion for this case. Table 1 gives the corresponding solution for a single focusing instrument when a smooth wire emitter is used as field ionization emitter [9]. The respective intensities at the times $t$ serve to determine $k(t)$ values defined as

$k(t)=\frac{\mathrm{d} i}{i_{0} \mathrm{~d} t}=\frac{\Delta i}{i_{0} \Delta t}$

where $i_{0}$ represents the intensity of the molecular ion at the time of ion formation and $\mathrm{d} i$ and $\mathrm{d} t$ are experimentally approximated by finite values $\Delta i$ and $\Delta t . \Delta i$ directly represents the measured intensity (corrected for reduced multiplier response) at mass position $m^{*}$. $\Delta t$ is obtained from the mass resolution of the instrument (defined at half peak height) as a rectangular 
TABLE 1

Conversion of mass to time scale for a single focusing instrument, smooth wire emitter

$t=\frac{2 X_{0}\left(\ln R / X_{0}\right)^{1 / 2}}{\left(2 e U / m_{1}\right)^{1 / 2}} \int_{0}^{y_{0}} \mathrm{e}^{y^{2}} \mathrm{~d} y$

with

$y_{0}=\left\{\frac{U-U_{x}}{U} \ln \left(R / X_{0}\right)\right\}^{1 / 2}=\left\{\frac{1-m^{*} / m_{2}}{1-m_{2} / m_{1}}\left(1-U_{G} / U\right) \ln \left(R / X_{0}\right)\right\}^{1 / 2}$

$X_{0}=$ wire radius $(\mathrm{cm})$.

$\boldsymbol{R}=$ distance emitter-counterelectrode (cm).

$U=$ potential difference emitter-counterelectrode (V).

$U_{x}=$ potential of position at which decomposition takes place.

$m^{*}=$ mass position to be converted to decomposition time.

$U_{\mathbf{o}}=$ potential counterelectrode.

$m_{1}=$ parent ion.

$m_{2}=$ fragment ion.

The integral is a function of the upper boundary alone.

approach. In a normal, i.e. low temperature, FI experiment, the intensity $i_{0}$ is often close to the molecular ion itself, which for stable ions represents by far the most intense peak in the spectrum. In principle, the parent and the fragment ion intensities integrated over the mass spectrometric time scale have to be summed to yield $i_{0}$. This is especially important when fragment ion intensities are large. More exact evaluations for this case are possible and have been described [10].

Relation between $k(t)$ and $k(E)$

The $k(t)$ measured in the FIK experiment is not yet a valuable piece of information. In order to learn more about its content, it is necessary to describe its relation to the unimolecular rate constant, $k(E)$, the basic quantity causing dissociation. Let us first assume that all parent ions possess the same amount of internal energy, $E$. The depletion of the parent ion intensity as a function of time then will be

$-\frac{\mathrm{d} M}{\mathrm{~d} t}=M k(E)$

And for a number of competing decay channels, the solution will be

$$
M=M_{0} \exp \left[-\sum_{j} k_{j}(E) t\right]
$$


Each channel $j$ contributes with its own $k_{j}(E)$. For the formation rate of one particular fragment, we obtain

$$
\frac{\mathrm{d} F}{\mathrm{~d} t}=M k(E)=k_{i}(E) M_{0} \exp \left[-\sum_{j} k_{j}(E) t\right]
$$

This equation holds for every particular internal energy if we assume that consecutive reactions do not take place. Let us now allow a distribution of internal energies for the precursor ion. In this case, we have to sum all individual rates described by $\mathrm{Eq}$. (5) with respect to internal energy. We obtain the integral form of Eq. (5) with $P(E)$ as the weighting function

$$
\frac{\mathrm{d} i}{i_{0} \mathrm{~d} t}=k(t)=\int_{0}^{\infty} P(E) k(E) \exp \left[-\sum_{j} k_{j}(E) t\right] \mathrm{d} E
$$

Here, the ion intensities $F$ and $M$ are replaced by the respective ion currents. This is justified when the detection probabilities for both are equal. Equation (6) gives the commonly known relation between $k(t)$ and $k(E)$. From Eq. (6), we can go back to considering only single internal energy ions. For these, the rate constants are not a function of energy. From Eq. (6), we have

$k_{E}(t)=k(E) \exp \left[-\sum k(E) t\right] \int_{E} P(E) \mathrm{d} E$

for each energy. The integral is termed $p_{E}$ in the following. For $p_{E}=1$, Eq. (7) is identical with Eq. (5). $p_{E}$ represents the fraction or relative weight of the particular energy in the total energy distribution. The $k(E)$ in the exponential expression is the parent ion depletion rate. For a single decomposition channel, relation (7) is illustrated in Fig. 2. The $P(E)$ function is represented by six $\delta$ functions. Three of them are below the threshold for decomposition, $E_{0}$. Hence $k(E)$ in Eq. (7) is zero. The other three $\delta$ functions serve to select a particular $k(E)$ from the continuous $k(E)$ function. Each $k(E)$ gives rise to one particular $k(t)$ function (dotted lines) given through relation (7). The total $k(t)$ observable in such a simplified case is just the sum over the individual $k_{E}(t)$

$k(t)=\sum_{E} k_{E}(t)=\sum_{E} k(E) \exp \left[-\sum_{j} k_{j}(E)\right] \cdot p_{E}$

This relation is represented by the solid line in the upper part of Fig. 2. The contribution of each $k(E)$ to the total $k(t)$ is strongest at $t=k(E)^{-1}$, as indicated by the dashed arrow lines. For $t<k(E)^{-1}$, larger $k(E)$ s dominate (note the $\log$ scale), while for $t>k(E)^{-1}$ the parent ions are depleted quickly. Figure 2 and Eq. (8) provide the basis for a numerical or step 


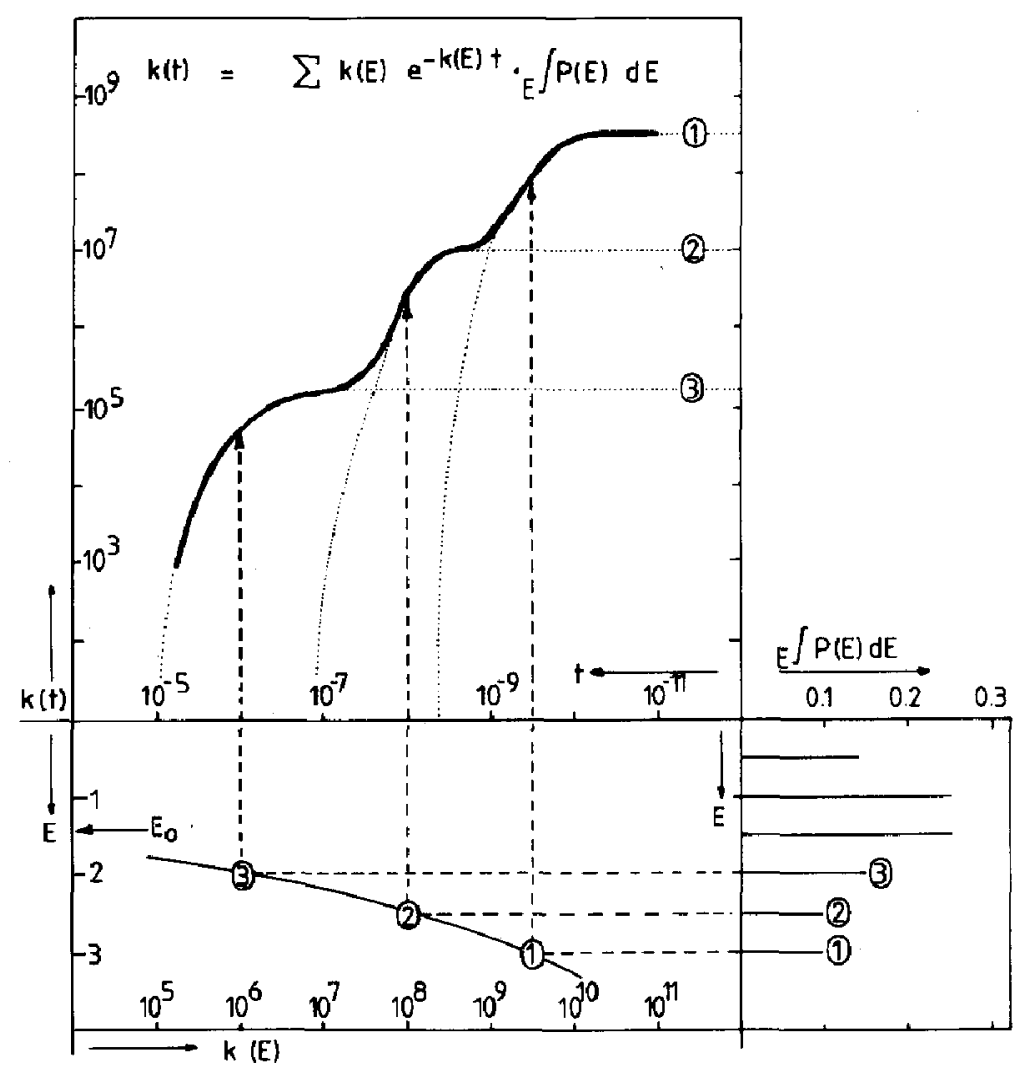

Fig. 2. Model calculation for monoenergetic parent ions to demonstrate the relationship between $P(E), k(E)$ and $k(t)$.

function approach to analyse experimental $k(t)$ functions in order to extract the unimolecular rate constant function $k(E)$.

Converting $k(t)$ vs. $t$ to $k(E)$ vs. $p_{E}$

In converting the experimental $k(t)$ vs. $t$ to a table of $k(E)$ vs. their contributions $p_{E}$, we assume that all parent ion depletion rates lead to fragmentation. Radiative relaxation to some state lower in energy than the lowest fragmentation threshold is excluded. For the extraction process, it is useful to sum up experimental $k(t)$ functions for one particular parent ion yielding

$$
\sum_{j} k(t)=\sum_{E} \sum_{j} k_{j}(E) \exp \left[-\sum_{j} k_{j}(E) t\right] \cdot p_{E}
$$

The advantage is that now the parent depletion rate $\Sigma_{j} k_{j}(E)$ is the only rate constant. Equation (9) can be rewritten to give

$k_{\text {depl }}(t)=\sum_{E} k_{\text {depl }}(E) \exp \left[-k_{\text {depl }}(E) t\right] \cdot p_{E}$ 
The individual rate constants $k_{j}(E)$ may be obtained later from the relation $\frac{k_{j}(t)}{k_{\mathrm{depl}}(t)}=\frac{k_{j}(E)}{k_{\mathrm{depl}}(E)}$

once $k_{\text {depl }}(E)$ is found. Relation (11) holds because the contribution $p_{E}$ of a particular set of rate constants $k_{\mathrm{j}}(E)$ is a property of the parent ion alone. Analysing the experimental data therefore proceeds as follows (see Fig. 3). The $k(t)$ point corresponding to the longest time observed experimentally $\left(t_{1}\right)$ has little contribution from larger $k(E)$ values (compare Fig. 2, energy $3)$. The corresponding parent ions have decomposed almost completely at shorter times. To a first approximation, we can identify the $k(E)$ responsible for the decomposition at this time with $t_{1}^{-1}$. This follows from the argument that the contribution of each $k(E)$ is strongest at $t=k(E)^{-1}$. From Eq. (10), we obtain

$k(t)=t^{-1} \exp (-1) \cdot p_{E}$

Hence, from experiment, we can deduce the contribution $p_{E}$ of the depletion rate $k(E)=t^{-1}$ to a first approximation if the $k(E)$ is taken to be uniform over the corresponding energy range. The numerical approach proceeds to shorter times in exactly the same way. Now, however, the contributions from rate constants $<t^{-1}$ at the time under investigation are subtracted first from the experimental $k(t)$ before determining the $p_{E}$ value. The analysis results in a table of $k(E)$ vs. $P_{E}$ values, i.e. a table of parent depletion rate constants active in the dissociation and their relative contributions $p_{E}$. The table is not yet very precise. However, accuracy can be gained from repeating the

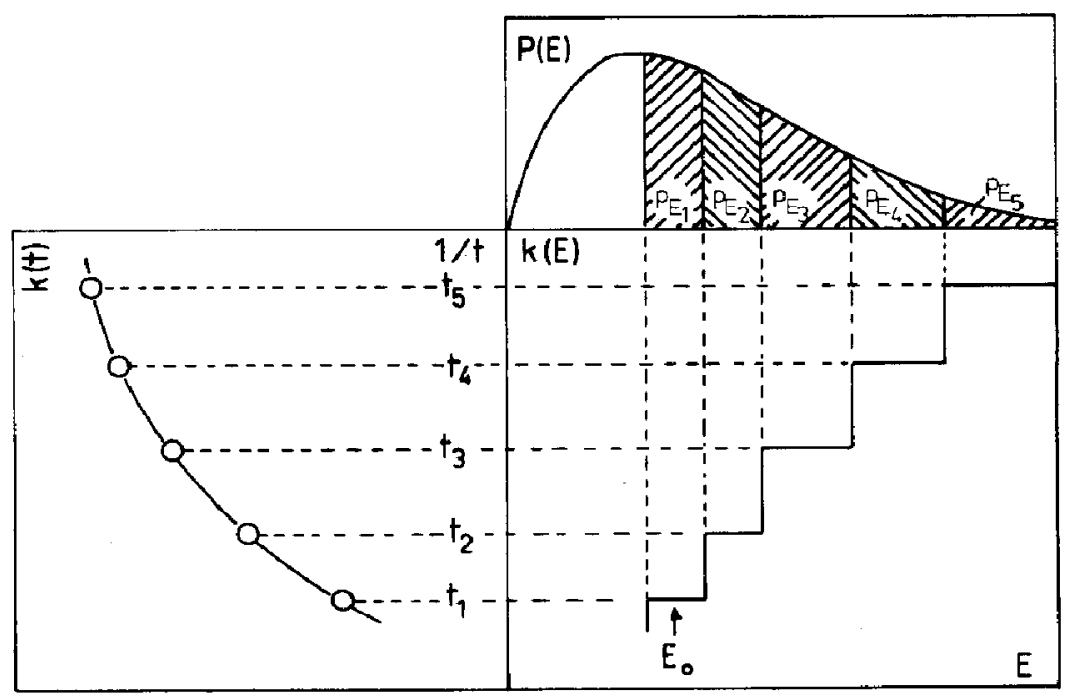

Fig. 3. Schematic representation to illustrate the step function procedure described in the text. 
analysis iteratively. The largest error is now contained in the first data point for the longest time. Here, no contribution from smaller $k(E)$ has been subtracted prior to analysis. The relative contribution is therefore too large. For this reason, this point is expelled from the table.

It is important to note that the table $k(E)$ vs. $p_{E}$ unambiguously and independently of any particular form of the parent ion internal energy distribution $P(E)$ shows which values of $k(E)$ have contributed to the experimentally observed fragmentation and to what extent.

Summing up the $p_{E}$ of all $k(E)$ values yields the area under the $P(E)$ function beyond the lowest dissociation threshold (see Fig. 3). This quantity shall be termed $Q$ in the following.

$Q=\int_{E_{0}}^{\infty} P(E) \mathrm{d} E$

This value $Q$ is an experimentally observable parameter; it represents the sum of all fragments (excluding surface and field dissociation products for which RRKM theory does not apply).

In principle, the $Q$ value obtained through the described analysis and the one measured directly should be identical. This, however, is not always the case. The reason is, on the one hand, that not all fragments are found at the detector, i.e. decompositions in the magnet, for instance, are missing. By far more important, on the other hand, is that the $k(t)$ may be slightly in error. Because of the uncertainty in the potential distribution between emittter and counterelectrode, especially when activated wires are used, and because of the rectangular approach, $\Delta t$ is the largest source of possible errors. Comparing the $Q$ values therefore provides a means for adjusting the experimental $k(t)$ to produce the correct total amount of fragmentation.

\section{Finding the energy correlation}

The problem of relating the $k(E)$ values to an appropriate internal energy scale can be solved only by making certain assumptions about the form of the energy distribution of the precursor ion. It is important that the assumptions are universally applicable because a detailed knowledge of the particulars of the $P(E)$ function is difficult to obtain. In a previous paper, we have described an experiment employing an isothermally heatable field ion source [11]. From the observed temperature dependence of the molecular ion abundance, we concluded that the amount of excess energy transferred during field ionization is small (several tenths of an eV). In this work, we assume that the internal energy distribution can be described by a smooth exponential function not exhibiting structure. This is consistent with previous findings [7], but the distribution may be more complicated as evidenced 
TABLE 2

Approximation functions for the internal energy distribution $P(E)$ of the precursor ion.

Eq. $P(E)$

no.
Evaluation of $\langle E\rangle$ (see text) ${ }^{\mathrm{a}}$

$$
\begin{aligned}
& \langle E\rangle=E_{0} / \ln (1 / Q) \\
& Q=\left[\sqrt{6 E_{0} /\langle E\rangle}+1\right] \mathrm{e}^{-\sqrt{6 E_{0} /\langle E\rangle}} \\
& Q=\left(2 E_{0} /\langle E\rangle+1\right) \mathrm{e}^{-\left(2 E_{0} /\langle E\rangle\right)} \\
& Q=\sqrt{\frac{6}{\pi\langle E\rangle}\left(1+\frac{2\langle E\rangle}{3 \sqrt{E_{0}}}\right) \mathrm{e}^{-\left(3 E_{0} / 2\langle E\rangle\right)}}
\end{aligned}
$$

For Eqs. (2)-(4), $\langle E\rangle$ is found using an iteration procedure.

by field ionization translational energy measurements [12]. We will come back to this point later.

Although it is expected that the details of the chosen function will affect the energy scale and hence the extracted $k(E)$ function, we will show that it is possible to obtain reliable information. The activation energy for the least energetic dissociation channel must be known for this purpose. The $P(E)$ functions used to create the $p_{E}-E$ correlation and hence to define the energy scale for the extracted $k(E)$ values are listed in Table 2. As an adjustable parameter, these $P(E)$ functions contain the average energy $\langle E\rangle$ defined by

$$
\langle E\rangle=\int_{0}^{\infty} E P(E) \mathrm{d} E
$$

The relationship between the parameter $\langle E\rangle$ and the experimental values $Q$ and $E_{0}$ is obtained from Eq. (12). The solutions of the corresponding integrals are also given in Table 2 . They serve to define the correct $\langle\mathrm{E}\rangle$ characterizing the $\mathrm{P}(\mathrm{E})$ function. It is important to note that the activation energy $E_{0}$ must include the kinetic shift for the lowest $k(E)$ in the table, i.e. the excess energy required to cause the parent to decompose with this particular rate constant. This means that, at best, the lower part of the rate constant function should be known already through another experiment (PEPICO, CE). Another approach is to calculate the lower part using RRKM theory, thereby imposing some uncertainty on the exact position of the extracted $k(E)$ s on the internal energy scale.

The four functions in Table 2 are smooth functions of energy. Because of the fact that decomposition occurs from the high energy tail of the distribution, the details at low energies (below the threshold for decomposition) are not critical. The essential difference between the functions is their slope above the threshold. 
The internal energy scale is now obtained by integrating the functions in Table 2 successively over the whole energy scale such that an area correspondence to the weight factors $p_{E}$ is created. This correspondence identifies the energy range for which a particular $k(E)$ is valid. The $k(E)$ function results as a series of steps (see Fig. 3).

\section{EXPERIMENTAL}

The halobenzene experiments were carried out on a ZAB-2f double focusing mass spectrometer equipped with a field ionization source without focusing lenses. FIK data were gained using a high voltage scan, the emitters were tungsten wires activated with benzonitrile $(10 \mu \mathrm{m}$ diameter before and $30 \mu \mathrm{m}$ after activation). In order to take the field of the whiskers in the potential distribution between emitter and counterelectrode into account, the modified distribution proposed in ref. 7 has been used to calculate the times and respective $\Delta t$ values. (For a treatment of the influence of the whiskers on the potential distribution of wire emitters, see ref. 33.)

The $t$-butylbenzene measurements were carried out on a modified MAT/CH-4 magnetic sector instrument equipped with an isothermally heatable field ionization source described in detail previously [7]. Before entering the electrostatic analyser, the ions were counted off axis using a pusher and a channeltron multiplier and sampling the spectra for 1-2 $\mathrm{h}$ in a multichannel analyser which was used as the master driving the magnet current. This was necessary to overcome the very low signal intensities when using $2 \mu \mathrm{m}$ diameter smooth tungsten wires as field ion emitters. The latter were manufactured using an electrochemical procedure similar to the one described by Goldenfeld et al, [34,35] to reduce the diameter of $10 \mu \mathrm{m}$ tungsten wires. All compounds used were high purity, commercially available samples.

\section{RESULTS AND DISCUSSION}

\section{Halogen loss from halobenzenes}

In order to test the outlined procedure and to find out if the $P(E)$ functions chosen are suitable to describe the experimental situation, we measured the $k(t)$ vs. $t$ functions for the halogen loss from the halobenzene radical cations. This system is particularly useful for several reasons.

(a) The unimolecular rate constants for the metastable region $\left(10^{4}-5 \times 10^{6}\right.$ $\mathrm{s}^{-1}$ ) have been determined by several authors now using different instruments [13-16]. Currently, halogen loss from halobenzenes seems to be the system which is understood best in the unimolecular dissociation of poly- 


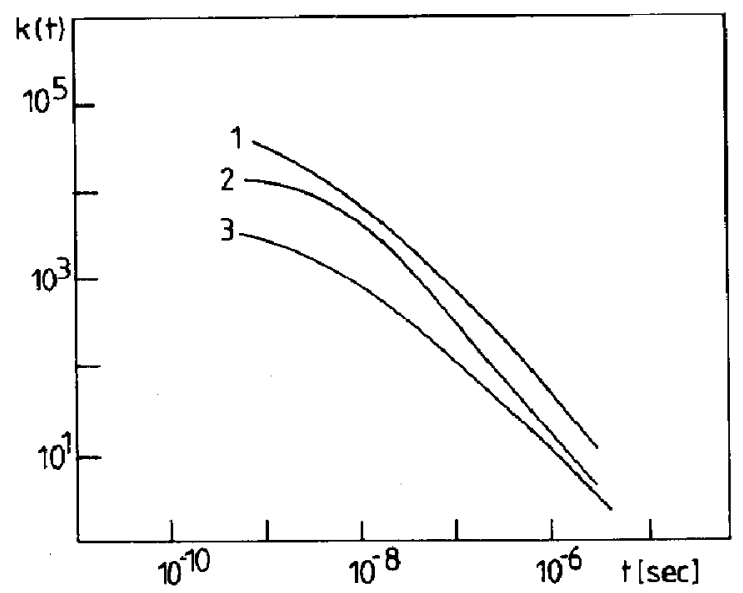

Fig. 4. $k(t)$ curves for halogen loss from halobenzenes. 1, Chlorobenzene; 2, iodobenzene; 3 , bromobenzene.

atomic ions. It has recently been proposed that the iodobenzene fragmentation represents a benchmark to be used in fragmentation rate measurements [17]. Burgers and Holmes [18] have used the known $k(E)$ vs. $E$ curves for halobenzene ion decomposition to define the threshold rate constant scale in appearance energy determinations from metastable decompositons. Durant et al. [19] employed chlorobenzene as a test case for their method of determining $k(E)$ from multiphoton ionization (MPI) data.

(b) The experimental rate constants are easily interpreted by RRKM calculations employing transition states for direct bond cleavages. Isomerization to a different ion structure prior to dissociation is therefore ruled out. This is confirmed by photodissociation (PD) experiments probing the nondecomposing ion structure. In these experiments, nice correlation between the photoelectron spectra of the neutrals and the PD spectra (= photoabsorption spectra) of the ions was observed, proving that the nature of the ions has not changed within milliseconds after ionization [20]. This is true in spite of the observed blue shift of the PD peaks, reflecting differences in the equilibrium geometries between the ground states of the neutral and the ion [20].

Figure 4 shows the $k(t)$ functions observed experimentally for halogen loss from the three halobenzenes studied. The $k(t)$ values are remarkably small and fall smoothly with time. The emitters were heated to approximately $800 \mathrm{~K}$ to cause sufficient fragmentation. It is noteworthy that, in the zero time spectra ${ }^{*}$, only chlorobenzene exhibits a very weak chlorine loss signal, while the metastable dissociation in the first field-free region is easily detectable for all three compounds. This is understood in terms of the low

* The "zero time spectra" contain the molecular ion, field-induced and surface products and the unimolecular decomposition products up to $10^{-11} \mathrm{~s}$. 
TABLE 3

Unimolecular rate constants, $k(E)$, and their relative contributions, $p_{E}$, extracted from the data in Fig. 4 using the outlined procedure

\begin{tabular}{|c|c|c|c|c|c|}
\hline \multicolumn{2}{|c|}{ Iodobenzene } & \multicolumn{2}{|c|}{ Bromobenzene } & \multicolumn{2}{|c|}{ Chlorobenzene } \\
\hline$k(E)$ & $p_{E}$ & $k(E)$ & $p_{E}$ & $k(E)$ & $p_{E}$ \\
\hline $3.1 \times 10^{9}$ & $1.1 \times 10^{-6}$ & $1.8 \times 10^{9}$ & $1.1 \times 10^{-6}$ & $1.0 \times 10^{9}$ & $2.4 \times 10^{-5}$ \\
\hline $3.6 \times 10^{8}$ & $3.0 \times 10^{-5}$ & $4.0 \times 10^{8}$ & $5.0 \times 10^{-6}$ & $1.8 \times 10^{8}$ & $6.2 \times 10^{-5}$ \\
\hline $1.0 \times 10^{8}$ & $5.3 \times 10^{-5}$ & $1.4 \times 10^{8}$ & $1.3 \times 10^{-5}$ & $3.1 \times 10^{7}$ & $1.1 \times 10^{-4}$ \\
\hline $2.9 \times 10^{7}$ & $3.4 \times 10^{-5}$ & $3.3 \times 10^{7}$ & $2.3 \times 10^{-5}$ & $1.0 \times 10^{7}$ & $5.3 \times 10^{-5}$ \\
\hline $1.0 \times 10^{7}$ & $3.6 \times 10^{-5}$ & $1.0 \times 10^{7}$ & $2.2 \times 10^{-5}$ & $3.1 \times 10^{6}$ & $6.8 \times 10^{-5}$ \\
\hline $2.5 \times 10^{6}$ & $2.7 \times 10^{-5}$ & $3.3 \times 10^{6}$ & $1.3 \times 10^{-5}$ & $5.6 \times 10^{5}$ & $9.3 \times 10^{-5}$ \\
\hline $5.6 \times 10^{5}$ & $2.6 \times 10^{-5}$ & $5.6 \times 10^{5}$ & $5.8 \times 10^{-5}$ & & \\
\hline
\end{tabular}

internal energy present in field ionized molecules compared with the very smoothly rising $k(E)$ values extracted from Fig. 4 and their relative contributions $p_{\mathrm{E}}$ to the dissociation observed (Table 3 ).

Figure 5 exhibits the extracted $k(E)$ functions when employing Eq. (2) in Table 2 for $P(E)$ to create the internal energy correlation. This function gave the best fit to the known slope of the $k(E)$ values. This, however, is no proof that the function (2) corresponds to the true energy distribution because there is a principle problem involved in this kind of gaining

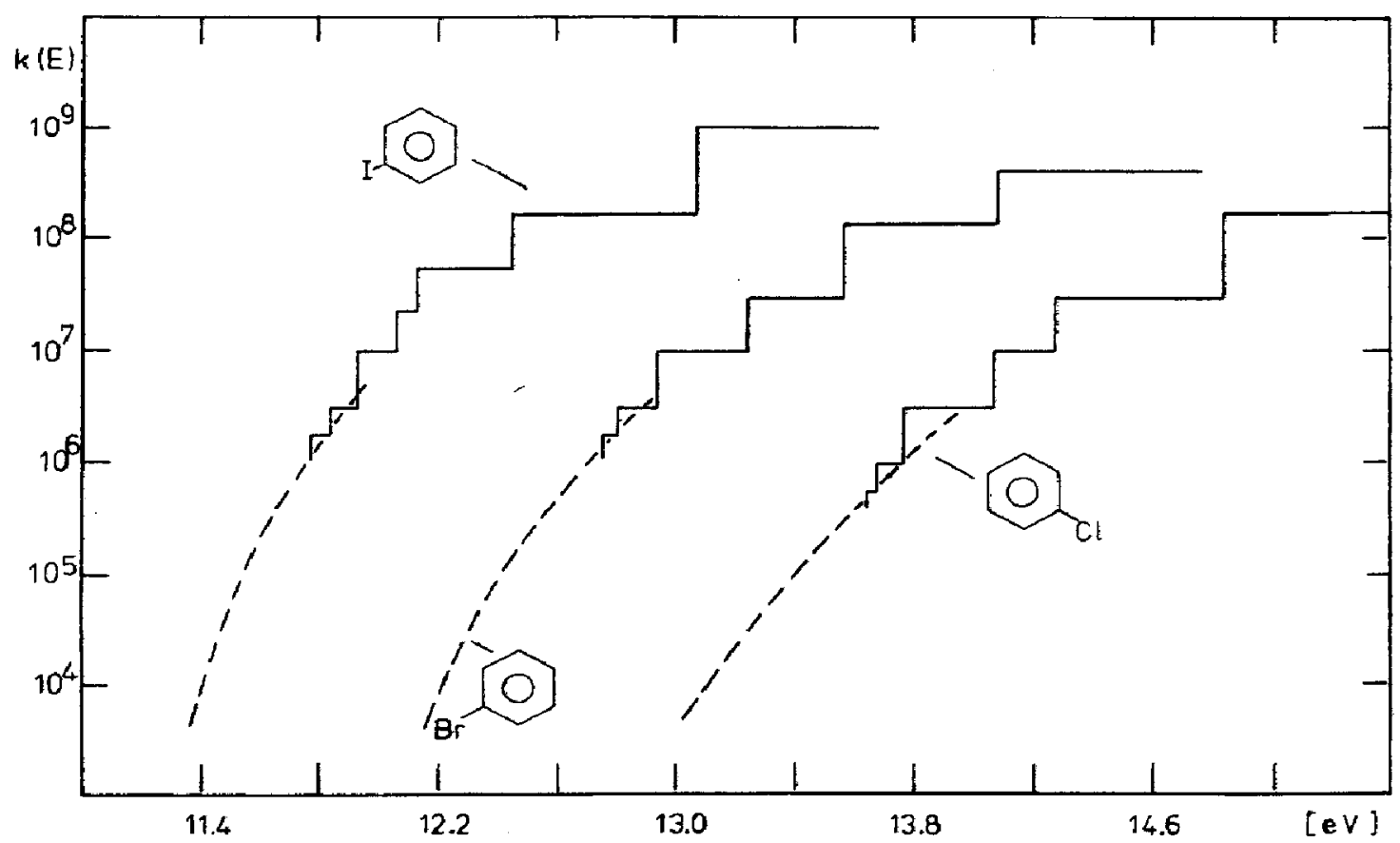

Fig. 5. $k(E)$ functions for halogen loss from halobenzenes. The energy correlation is found by assuming Eq. (2) in Table 2 to be $P(E)$ function. PEPICO results from ref. 13. 
information on the rate vs. energy functions. The amount of fragmentation for the halobenzenes did not exceed $5 \times 10^{-4}$ of the total ion current observed at the detector. This is a situation most frequently encountered in field ionization. The molecular ion is by far the most intense peak in the spectrum,. i.e. the internal energy distribution has its maximum far below the lowest dissociation threshold; field ionization is a so-called "sof $t$ " ionization method. Therefore the dynamic information in FIK is gained from the high energy tail of the internal energy distribution (actually, the amount of fragmentation shown schematically in Fig. 3 is exaggerated for most practical cases). The energies shown in Fig. 5 should therefore not be confused with any average energy content of the ions prior to dissociation. Only a negligible part of the ions possess enough energy to decompose at all. Similarly, Eq. (2) in Table 2 fulfils the requirement of a good simulation of the $P(E)$ function only above threshold. It may be seriously in error below this value.

\section{Methyl loss from t-butylbenzene}

The second process studied is the methyl loss from $t$-butylbenzene radical cations. This process has been studied frequently by electron impact [21-23], field ionization [7,24,25], PEPICO [26] and photodissociation [27]. It seems to be an ideal case because there is only one decomposition channel in the low energy range. In addition, the process is easily detectable as a time-delayed dissociation in field ionization.

As already found in a previous experiment [7] the $k(t)$ function again exhibits a change in slope between short and longer times at about $5 \times 10^{-9}$ $s$ (Fig. 6).

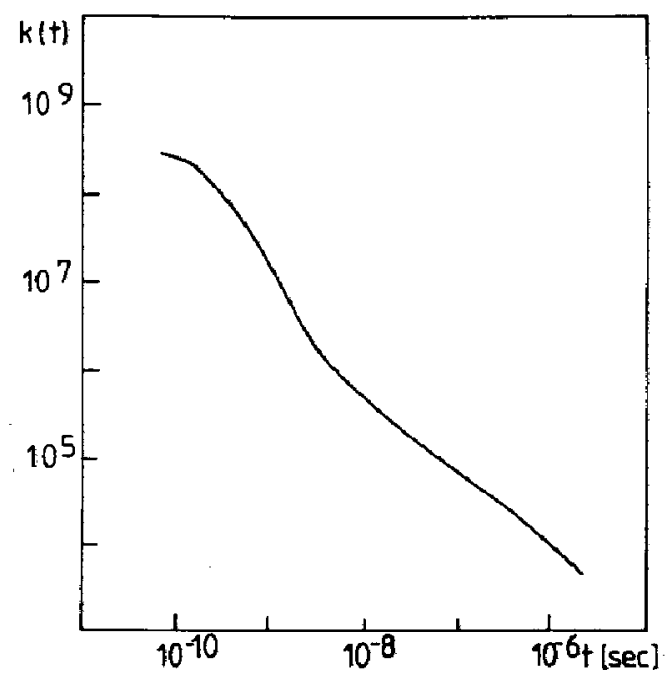

Fig. 6. $k(t)$ function for methyl loss from $t$-butylbenzene molecular ion. 
In order to eliminate uncertainties in the potential distribution we used 2 $\mu \mathrm{m}$ diameter wires as field anodes. The break in the $k(t)$ function correlates well with a sudden change in the secondary isotope effect $\left(\mathrm{CH}_{3}\right.$ vs. $\mathrm{CD}_{3}$ loss $)$ from about 1 at short times to 2 at longer times as observed by van der Greef [25] using FIK. This observation has not found a sound explanation so far. A time dependence of this isotope effect has also been observed in the field-free regions of a double focusing mass spectrometer following EI ionization [22].

Figure 6 shows the experimental $k(t)$ function measured at $510 \mathrm{~K}$ employing the isothermally heatable FI source described previously [7,10]. From this curve, the step functional $k(E)$ curve in Fig. 7 has been derived using the procedure outlined above. The $P(E)$ function used to create the energy correlating for the $p_{E}$ values was of the same type as in the halobenzene analysis [Eq. (2) in Table 2] with the proper characteristic value $Q$ from our experiment. The PEPICO results from ref. 26 are also shown for comparison. They were used to identify the correct position of the lowest $k(E)$ on the internal energy scale. The break in the $k(t)$ function finds a correspondence in the extracted $k(E)$. The experimental step function curve systematically underestimates the extrapolated PEPICO based $k(E)$ function between 1.8 and $2.2 \mathrm{eV}$ excess energy.

There are, in principle, two explanations for such an experimental observation: one is that there is more than one dissociation channel leading to the fragment ion $\mathrm{C}_{9} \mathrm{H}_{11}^{+}$and the other is that $P(E)$ is not a smooth function of energy. Both cases shall be discussed in greater detail in the following.

First case. Suppose the following reaction sequence is responsible for the

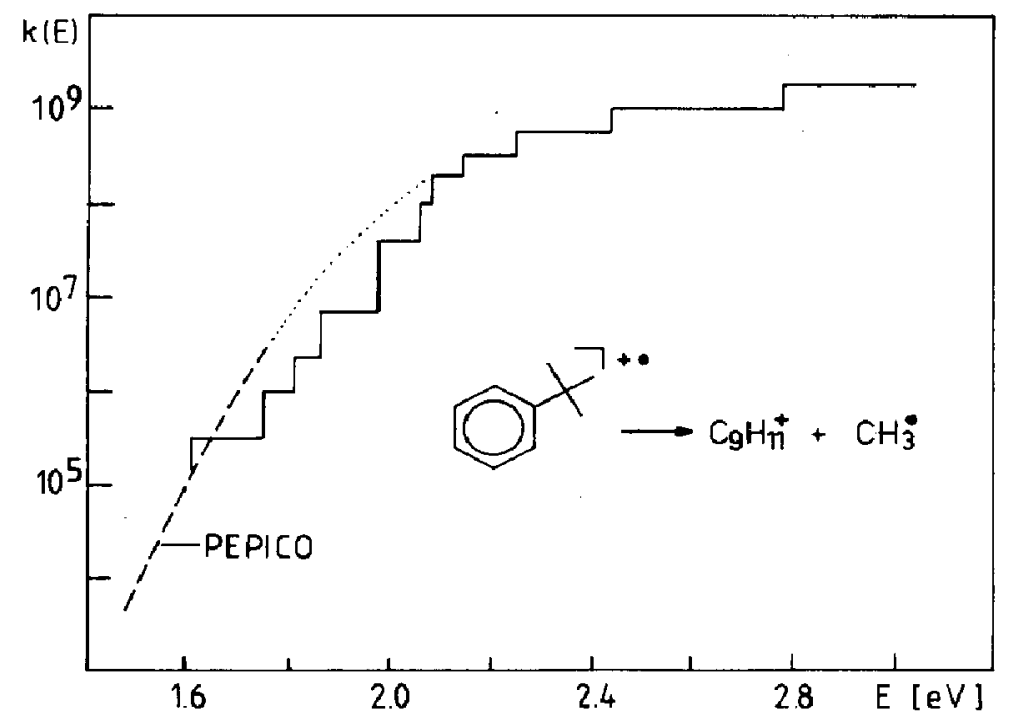

Fig. 7. $k(E)$ function for methyl loss from $t$-butylbenzene molecular ion. 
observed formation of $\mathrm{C}_{9} \mathrm{H}_{11}^{+}$ions, the $k_{i}$ being the individual unimolecular rate constants $k(E)$.

$\mathrm{m}_{1}^{+\cdot} \stackrel{\mathrm{k}_{1}}{\longrightarrow} \mathrm{C}_{9} \mathrm{H}_{11}^{+}+\mathrm{CH}_{3}^{*}$

$\mathrm{m}_{2}^{+*} \stackrel{k_{3}}{\longrightarrow} \mathrm{C}_{9} \mathrm{H}_{11}^{+}+\mathrm{CH}_{3}^{*}$

For $k_{2}=0$, a $k(t)$ as shown in Fig. 8 as the dotted line 1 would result for monoenergetic $\mathrm{m}_{1}^{+}\left(p_{E}=1\right)$. However, if $k_{2}$ is of the same order of magnitude or larger than $k_{1}$, it is responsible for a much faster depletion of $\mathrm{m}_{1}^{+}$, shifting line 1 into the solid line $2 . \mathrm{m}_{2}^{+}$might further decompose with a slower rate constant, $k_{3}$, yielding the same fragment $\mathrm{C}_{9} \mathrm{H}_{11}^{+}$. The $k(t)$ observable in this (monoenergetic) case would be a superposition of lines 2 and 3. Such a situation may hold for a wider range of energies such that a break in $k(t)$ in a real (polyenergetic) case is observed. However, for this situation to occur, it is necessary that the ion $\mathrm{m}_{2}^{+}$is the preferred structure of the parent ion or, in other words, that it is lower in energy. In addition, there must be an open isomerization pathway to this structure below the threshold for decomposition. The consequences of this scenario would be that the extracted $p_{E}$ values are in error. The basis for the letter is that one is dealing with the true parent ion depletion rate. This rate includes the isomerization rate constant $k_{2}$. Hence, the $p_{E}$ are in error when $k_{2}$ is not known.

For $t$-butylbenzene, it is unlikely that the parent isomerizes to a structure

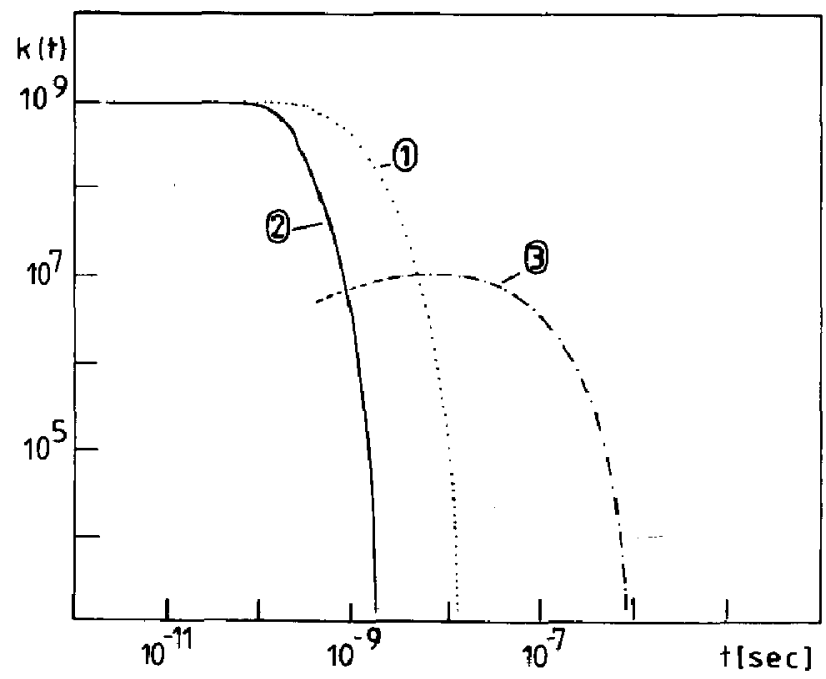

Fig. 8. Schematic representation of $k(t)$ functions for monoenergetic parent ions, when isomerization is involved (see text). $1, k(t)=k_{1} \mathrm{e}^{-k_{1} t}, k_{1}=10^{9} ; 2, k(t)=k_{1} \mathrm{e}^{-\left(k_{1}+k_{2}\right) t}, k_{2}=5$ $\times 10^{9} ; 3, k(t)=k_{3} \mathrm{e}^{-k_{3^{t}}}, k_{3}=10^{6}$. 
much lower in energy prior to decomposition. Another possibility is that competition between isomerization and dissociation due to different internal energies of the precursor takes place. For instance, direct dissociation from the original ion structure is favoured at high internal energies while, at lower energies, isomerization is necessary, leading to a different parent ion. This then dissociates to form fragments of the same elemental composition but different structure. In such a case, the extracted $p_{E}$ are correct and the break in the $k(t)$ function stems from a break in the $k(E)$ curve for the parent ion depletion. A possible reaction mechanism would be

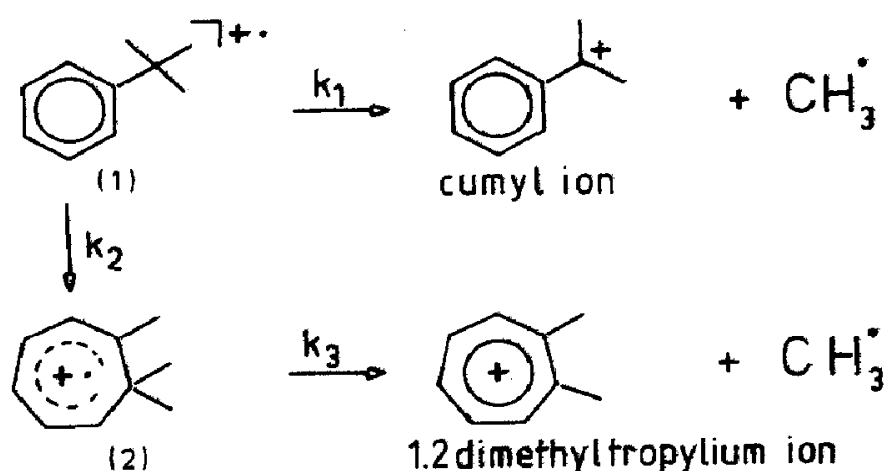

In this scheme, (1) is the lower parent ion structure while, for the fragment ions, the tropylium-type structure may be lower in energy than the cumyl structure. Information about the energetics is gained from the homologue tropylium and methyltropylium ions and their respective benzyl counterparts. For tropylium/benzyl the difference in the heats of formation is $\mathbf{1 5}$ $\mathrm{kcal} \mathrm{mol}^{-1}$ [28]. The addition of the methyl groups will stabilize the benzyl structure more than the tropylium ion, which gains its stability from its aromaticity. The $\Delta H_{\mathrm{f}}$ difference between the two $\mathrm{C}_{9} \mathrm{H}_{11}^{+}$ions in Scheme 2 is therefore believed to be small. It is difficult to decide which of the two is more stable. If we assume that the tropylium structure is more stable, dissociation at low energies is possible via the 7-membered ring only following 1 to 2 methyl migration. At slightly higher energies, the direct dissociation leading to the cumyl ion becomes fast enough to suppress this channel completely.

The proposed reaction Scheme 2 can be tested by a simple experiment. The $\mathrm{C}_{9} \mathrm{H}_{11}^{+}$ions formed in high energy dissociations should be different from those formed, for instance, in the field-free regions of a sector mass spectrometer. We have carried out a collision-induced dissociaton (CID) experiment using the $\mathrm{C}_{9} \mathrm{H}_{11}^{+}$ions from a conventional $70 \mathrm{eV}$ EI source as a precursor and comparing its dissociation with the one obtained from the metastable $\mathrm{C}_{9} \mathrm{H}_{11}^{+}$formed in the first field-free region. After correction for the metastable $\mathrm{C}_{2} \mathrm{H}_{4}$ loss, the spectra were identical within experimental 
error. Unfortunately, we could not take a special look at methyl loss because of interference with an artifact peak at this position. Photodissociation experiments [27] in an ICR cell suggest that the $\mathrm{C}_{9} \mathrm{H}_{11}^{+}$ion formed following $12 \mathrm{eV}$ EI ionization has the cumyl structure. However, no other structure has been discussed in the paper. Köppel and McLafferty [29] studied the CID spectra of $\mathrm{C}_{9} \mathrm{H}_{11}^{+}$ions formed from a variety of precursors and found a total number of 13 different ion structures. In the particular case of $t$-butylbenzene as a precursor, no dependence of the CID spectrum on the impact energy of the electrons could be detected.

On the basis of the experiments, we are left with the conclusion that the proposed reaction Scheme 2 cannot be ruled out completely, but that it is not very likely either.

Another interpretation of the $t$-butylbenzene data based on reaction Scheme 1 is competition between direct dissociation from the side-chain ionized parent ion and decomposition following intramolecular relaxation to the (hot) ionic ground state. This, however, would mean that the ionic ground state with an onset near $10.3 \mathrm{eV}$ in the photoelectron spectrum [30] survives at least $10^{-9} \mathrm{~s}$ before relaxing to the $X$ state or suffer radiative decay to the (cold) ground state. In the light of the rate constants common in radiationless intramolecular relaxation (see, for instance, ref. 31), this explanation seems to be rather unlikely in $t$-butylbenzene ion decomposition.

Second case. The basis for the correlation between the $p_{E}$ values extracted from the experimental $k(t)$ function and the internal energy scale is the assumption of a smooth $p(E)$ function (Table 2). As already mentioned, there is experimental evidence that the energy deposition function in field ionization exhibits considerable structure even for larger molecular ions. Jason and Parr [12] measured high resolution field ion energy spectra of toluene, cycloheptatriene and phenol. In all three cases, a characteristic structure was observed which could, in part, be related to photoionization and photoelectron spectra. Although the quantifative agreement is poor, one can arrive at the conclusion that the energy deposition function may be represented by a convolution of Franck-Condon factors and the tunneling probability for field ionization. The latter discriminates strongly against higher ionization energies (see, for instance, ref. 32) with the consequence that most parent ions are formed close to the ground state. In $t$-butylbenzene, there is a large gap between the first ionization potential at $8.7 \mathrm{eV}$ and a series of excited states with an onset near $10.3 \mathrm{eV}$ [30]. The latter corresponds to side-chain ionization and is about $0.4 \mathrm{eV}$ above the threshold for methyl loss [26]. In the light of these considerations, the break in the $k(t)$ curve may stem from enhanced population of states above $10.3 \mathrm{eV}$ compared with the smooth $P(E)$ function assumption. The systematic underestimation of the $k(E)$ curve between 1.8 and $2.2 \mathrm{eV}$ is then an artifact of the energy correlation procedure. 
Both the cases discussed, reaction Scheme 2 and a structured $P(E)$ function, are able to explain the observed dependence of the secondary isotope effect on time. In the latter case, the sudden increase if the $m$ $\mathrm{CH}_{3} / m-\mathrm{CD}_{3}$ ratio is due to a $P(E)$ function with a peak near $10.3 \mathrm{eV}$ and therefore an artifact of the ionization method. There is no change in reaction mechanism. It should be pointed out that the sudden change observed by van der Greef [25] would be smoothed out if the measurements were carried out at higher temperatures $(>650 \mathrm{~K})$. Then, the $P(E)$ function will still exhibit structure but the gaps in between are filled such that the smooth function approach for $P(E)$ will become a better approximation [compare also the procedure in ref. 7 where only the high temperature measurements were taken as a basis for the $\mathrm{k}(E)$ extraction].

In the realm of Scheme 2, the secondary isotope effect is understood in the following way: The first step, isomerization to $\mathrm{m}_{2}^{+}$, is obviously well above threshold when looking on ions with just enough energy to decompose. The migration rates for both $\mathrm{CH}_{3}$ and $\mathrm{CD}_{3}$ should be about equal. This step is not responsible for the secondary isotope effect. For the dissociation step, however, leading to the 1,2 dimethyltropylium ion, it is expected that, close to threshold, $\mathrm{CH}_{3}$ is lost preferentially to $\mathrm{CD}_{3}$ because of the higher density of states for the ion with the $\mathrm{CD}_{3}$ group intact. Zero point energy differences point in the same direction.

Because of the experimental observation that the partial CID spectra for the $\mathrm{C}_{9} \mathrm{H}_{11}^{+}$ions from the source and from the first field-free region are very similar and because of the other experimental evidences discussed, we favour the explanation of a structured $P(E)$ function over reaction Scheme 2. A decisive experiment would be to monitor the field ion energy distribution of the parent ion using high resolution. It is expected that structure is observed near $10.3 \mathrm{eV}$ assuming that the onset is defined by the ionization energy of $8.7 \mathrm{eV}$.

\section{CONCLUSION}

It has been demonstrated that FIK can be used to evaluate approximate $k(E)$ functions in orders of magnitude $>10^{7} \mathrm{~s}^{-1}$ if the parent ion depletion rate is completely observable in the fragment ions. It is believed that this is true for most applications in the unimolecular dissociations of ions. Exceptions are expected for ions suffering strong radiative decay and for ions which undergo competition between isomerization and dissociation at one and the same internal energy. FIK, in principle, is not able to distinguish between this case, which can only be detected employing internal energy selection, and an apparent competition between isomerization and dissociation based on different internal energies of the respective precursors. For the 
halogen loss from halobenzene molecular ions, the agreement between the numerical approach presented and the well known energy dependence of the unimolecular rate constant $k(E)$ is satisfactory within experimental uncertainty. This can be taken as a justification for the smooth function approach to the internal energy distribution $P(E)$ in this case.

For methyl loss from the $t$-butylbenzene ion, the experimental break in the $k(t)$ function is probably due to a structured $P(E)$ function rather than to a change in reaction pathway at low internal energies. It is recommended that work is carried out at high temperatures $(600-900 \mathrm{~K})$ to smooth out the internal energy distribution and thus justify the use of a smooth $P(E)$ function.

The numerical approach presented in this study is a useful simplification for the analysis of FIK data and can be installed easily if computers are available. The drawbacks of the method are mainly the uncertainties in the functional form of the high energy tail of the parent ion internal energy distribution and the need for supportive $k(E)$ data near the threshold for decomposition.

\section{ACKOWLEDGEMENTS}

We would like to thank Ron Hass for the opportunity to carry out the halobenzene measurements on his ZAB instrument. For helpful discussions, we are grateful to Steen Hammerum and Tom Baer. In a special way, we are indebted to Karsten Levsen for numerous discussions and for the opportunity to carry out this work in his laboratory. Financial support from the Deutsche Forschungsgemeinschaft is kindly acknowledged.

\section{REFERENCES}

1 R.A. Marcus and O.K. Rice, J. Phys. Colloid. Chem., 55 (1951) 894.

2 P.J. Robinson and K.A. Holbrook, Unimolecular Reactions, Wiley-Interscience, London, 1972.

3 H.M. Rosenstock, M.B. Wallenstein, A.L. Wahrhaftig and H. Eyring, Proc. Natl. Acad. Sci. U.S.A., 38 (1952) 667.

4 C. Lifshitz, Adv. Mass Spectrom., 7A (1978) 3; Mass Spectrom. Rev., 1 (1982) 309.

5 T. Baer, in M.T. Bowers (Ed.), Gas Phase Ion Chemistry, Vol. 1, Academic Press, New York, 1979.

6 B. Andlauer and C. Ottinger, J. Chem, Phys., 55 (1971) 1471.

7 W.A. Brand and K. Levsen, Int. J. Mass Spectrom. Ion Phys., 51 (1983) 135.

8 H.D. Beckey, Z. Naturforsch. Teil A, 19 (1964) 71; 17 (1962) 1103.

9 K. Levsen, Habilitation Thesis, Bonn, 1974.

10 W. Brand, H.D. Beckey, B. Fassbender, A. Heindrichs and K. Levsen, Int. J. Mass Spectrom, Ion Phys., 35 (1980) 11.

11 W. Brand and K. Levsen, Int. J. Mass Spectrom. Ion Phys., 35 (1980) 1. 
12 A.J. Jason and A.C. Parr, Int. J. Mass Spectrom. Ion Phys., 22 (1976) 221.

13 T. Baer, B.P. Tsai, D. Smith and P.T. Murray, J. Chem. Phys., 64 (1976) 2460.

14 T. Baer and R. Kury, Chem. Phys. Lett., 92 (1982) 659.

15 H.M. Rosenstock, R. Stockbauer and A.C. Parr, J. Chem. Phys., 71 (1979) 3708.

16 H.M. Rosenstock, R. Stockbauer, and A.C. Parr, J. Chem. Phys., 73 (1980) 773.

17 J. Dannacher, H.M. Rosenstock, R. Buff, A.C. Parr, R.L. Stockbauer, R. Bombach and J.P. Stadelmann, Chem. Phys., 75 (1983) 23.

18 P.C. Burgers and J.L. Holmes, to be published (1984). See also J.L. Holmes, 31st ASMS Conf., Boston, 1983, p. 666.

19 J.L. Durant, D.M. Rider, S.L. Anderson, F.D. Proch and R.N. Zare, J. Chem. Phys., (1984) in press.

20 R.C. Dunbar, H.H.-I. Teng and E.W. Fu, J. Am. Chem. Soc., 101 (1979) 6506.

21 P.N. Rylander and S. Meyerson, J. Am. Chem. Soc., 78 (1956) 5799.

22 R. Neeter and N.M.M. Nibbering, Org. Mass Spectrom., 7 (1973) 1091.

23 N.A. Ucella and D.H. Williams, J. Am. Chem. Soc., 94 (1972) 8778.

24 G. Tenschert and H.D. Beckey, Int. J. Mass Spectrom. Ion Phys., 7 (1971) 97.

25 J. van der Greef, Ph.D. Thesis, Amsterdam, 1980.

26 W.A. Brand and T. Baer, Int. J. Mass Spectrom. Ion Phys., 49 (1983) 103.

27 Y. Takenoshita and R.C. Dunbar, Bull. Chem. Soc. Jpn., 53 (1980) 2185.

28 R. Bombach, J. Dannacher and J.P. Stadelmann, Chem. Phys. Lett., 95 (1983) 259.

29 F.W. McLafferty, private communication, 1983.

30 D.W. Turner, C. Baker, A.D. Baker and C.R. Brundle, Molecular Photoelectron Spectroscopy, Wiley-Interscience, New York, 1970.

31 (a) J.P. Maier, in P. Ausloos (Ed.), Kinetics of Ion-Molecule Reactions, Plenum Press, New York, 1979, p. 437.

(b) T.A. Miller, Ann. Rev. Phys. Chem., 33 (1982) 257.

(c) J.P. Maier, O. Morthaler and M. Mohraz, J. Chim. Phys., 77 (1980) 661.

32 R. Gomer, Field Emission and Field Ionization, Harvard University Press, Cambridge, MA, 1961.

33 K. Levsen, E. Hilt and I. Goldenfeld, Int. J. Mass Spectrom. Ion Phys., 51 (1983) 347.

34 I.V. Goldenfeld and K. Weil, Z. Metallkd., 73 (1982) 190.

35 W.A. Schmidt, I.V. Goldenfeld and A.I. Helal, Int. J. Mass Spectrom. Ion Phys., 38 (1981) 241. 